\title{
Searches for Beyond nuSM Physics with MINOS/MINOS+
}

\section{Adam Schreckenberger*}

University of Texas at Austin, Department of Physics, 2515 Speedway, C1600, Austin, TX

78712-1192

E-mail: adamsephysics .utexas.edu

\section{Stefano Germani}

Department of Physics and Astronomy, University College London, London WC1E 6BT, United Kingdom

E-mail: s.germani@ucl.ac.uk

The MINOS+ experiment is an on-axis neutrino oscillation search situated in the Fermilab NuMI beam. We utilize $v_{\mu} \rightarrow v_{e}$ appearance candidates to probe for exotic neutrino oscillation phenomena. Here we consider a $3+1$ sterile neutrino model, where $v_{\mu} \rightarrow v_{e}$ oscillation could be further mediated by the presence of a sterile neutrino, to place limits upon $\sin ^{2} 2 \theta_{\mu e}$ at values of $\Delta m_{41}^{2}<1$ $\mathrm{eV}^{2}$ using the first year of MINOS+ data. The results of this analysis are compared to those of LSND and MiniBooNE.

38th International Conference on High Energy Physics 3-10 August 2016

Chicago, USA

${ }^{*}$ Speaker. 


\section{Introduction}

MINOS+ represents the natural extension of the Main Injector Neutrino Oscillation Search (MINOS) in a medium-energy NuMI beam configuration that peaks around $6 \mathrm{GeV}$ [1]. MINOS+ consists of two functionally identical steel-scintillator tracking calorimeters: a $0.98 \mathrm{kt}$ Near Detector (ND) and a $5.4 \mathrm{kt}$ Far Detector (FD). The ND is located at Fermilab at a baseline of $1.04 \mathrm{~km}$, whereas the FD is in the Soudan Mine in Northeastern Minnesota at a baseline of $735 \mathrm{~km}$.

The shift of the beam peak to a higher energy with respect to that of MINOS makes MINOS+ an ideal probe for potential exotic oscillation mechanisms. LSND and MiniBooNE both observed $v_{e}$ and $\bar{v}_{e}$ appearance rates that were inconsistent with standard three-flavor neutrino oscillation [2, 3]. A potential explanation for these results is the introduction of sterile neutrinos, which do not couple to the weak vector bosons but could still impact the oscillation formalism. In a 3+1 model, the probability of a $v_{\mu} \rightarrow v_{e}$ transition in vacuum is expressed as:

$$
\begin{gathered}
P\left(v_{\mu} \rightarrow v_{e}\right)=\Sigma_{i>j}\left\{-4 \Re\left(U_{\mu i}^{*} U_{e i} U_{\mu j} U_{e j}^{*}\right) \sin ^{2}\left(\Delta_{i j}\right)+2 \mathfrak{I}\left(U_{\mu i}^{*} U_{e i} U_{\mu j} U_{e j}^{*}\right) \sin \left(\Delta_{i j}\right)\right\} \approx \\
\sin ^{2}\left(2 \theta_{13}\right) \sin ^{2}\left(\theta_{23}\right) \sin ^{2}\left(\Delta_{31}\right)+\sin ^{2}\left(2 \theta_{14}\right) \sin ^{2}\left(\theta_{24}\right) \sin ^{2}\left(\Delta_{41}\right)+f\left(\delta_{13}, \delta_{24}-\delta_{14}, \theta_{i j}\right),
\end{gathered}
$$

where the $U$ 's are PMNS matrix elements, $\Delta_{i j} \propto m_{i}^{2}-m_{j}^{2}$, and $f$ covers additional terms.

The introduction of a sterile neutrino adds three new Euler angles and two new CP-violating phases. In particular, this analysis is sensitive to $\theta_{14}$ and $\theta_{24}$. However, there are additional dependencies that need to be considered, including the three-flavor parameters $\Delta m_{32}^{2}, \theta_{13}, \theta_{23}$, and $\delta_{13}$; as well as the sterile parameters $\theta_{34}$ and $\delta_{24}-\delta_{14}$.

We present our preliminary results on the first year of MINOS+ data, corresponding to an exposure of $2.97 \times 10^{20}$ protons-on-target (POT). Two additional years of data are left to analyze.

\section{Event Selection and Cross Checks}

MINOS+ makes use of a Library Event Matching (LEM) algorithm to select $v_{e}$ Charged Current (CC) event candidates in the detectors. This vetted technique was utilized in previous MINOS analyses and returns a single value discriminant, $\left(\alpha_{L E M}\right)$, by means of a large library of simulated FD Monte Carlo and an artificial neural network [4]. The library consists of Neutral Current (NC) and $v_{e}$ CC events.

Log-likelihood comparisons between input candidate and library topologies are performed and ranked. Information from the 50 best library matches is fed to the artificial neural network to produce the final discriminant. The input variables include: 1) the fraction of best 50 events that are $v_{e} \mathrm{CC}$ events; 2) the mean inelasticity of $v_{e} \mathrm{CC}$ events in the best 50;3) the mean matched charge of $v_{e} \mathrm{CC}$ events in the best 50; and 4) the reconstructed energy of the input candidate.

The LEM selector yields a clear shape difference between signal $v_{e} \mathrm{CC}$ and background events. The additional cut on reconstructed energy between $6-12 \mathrm{GeV}$ focuses the analysis on a range suitable for exotic oscillation searches and removes a significant percentage of background events in the data sample. For this study, we cut on $\alpha_{L E M}>0.6$ to produce our signal-selected region.

Prior to making the fit, several sideband checks are performed to validate the prediction method and LEM selector employed in the analysis. First, we look at events with $\alpha_{L E M}<0.5$. This AntiPID check compares the three-flavor prediction to the data in a range where no $v_{e}$ excess is expected. 
We demand that the AntiPID prediction be statistically indistinguishable from the FD data. We observe 62 AntiPID events in data and predict $64.5 \pm 8.0_{\text {stat }}$ events.

Given that this is a background-dominated analysis, additional care regarding the assessment of NC events in the $\alpha_{L E M}>0.6$ region is taken. A Muon Removed Charged Current (MRCC) method is used to create NC-like samples from $v_{\mu} \mathrm{CC}$ data and Monte Carlo. We require a less than $2 \sigma$ discrepancy in this sideband. 59 events were observed in the FD MRCC data, and 51.6 \pm 7.0 events were predicted in the sample.

\section{Results}

78 events were observed in the LEM-selected FD data during the first year of MINOS+. Compared to the 56.7 predicted events, this represents a $2.3 \sigma$ excess when including statistical and systematic errors. The comparisons of various FD energy spectra are shown in Figure 1. The largest sources of the FD data excess in the energy spectrum do not fit well with either the threeflavor or 3+1 model predictions; further analysis on two additional years of data will help elucidate if MINOS+ is observing upward statistical fluctuations. Nonetheless, the picture provided by the energy distribution is incomplete. We additionally use the LEM shape information to perform a fit in the 3+1 parameter space. An 18-bin fit, composed of 3 bins of $\alpha_{L E M}$ and 6 bins of reconstructed energy, was chosen for this analysis based upon sensitivity considerations, and systematic errors were included as a covariance matrix.

The MINOS+ $v_{e}$-driven sterile neutrino search provides a complimentary study to the combined MINOS/Daya Bay/Bugey-3 result. This is a robust study that includes considerations of all of the added $3+1$ model parameters. The $v_{e}$ contour shown in Figure 2 was produced from log-likelihood surfaces spanning $\theta_{14}$ and $\theta_{24}$ at various values of $\Delta m_{41}^{2}$. The $\theta_{34}, \delta_{13}$, and $\delta_{24}-\delta_{14}$ parameters were profiled for each of these slices. The three-flavor oscillation parameters, excluding $\delta_{13}$, were fixed to the global best values.

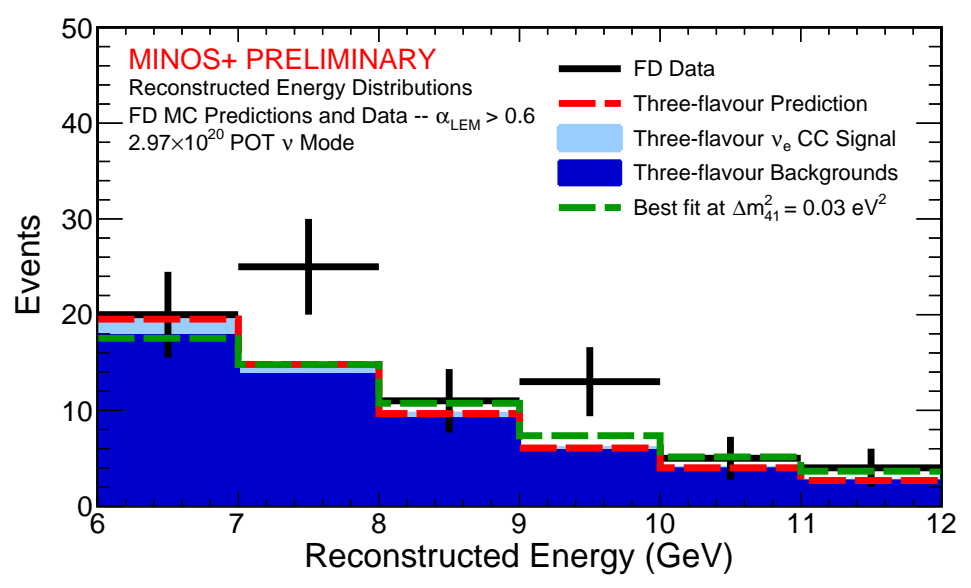

Figure 1: FD energy spectra for signal-selected data, the three-flavor background and signal predictions, and a $3+1$ sterile model prediction. $\alpha_{L E M}>0.6$ is required for all events, and global best values were used to perform the three-flavor predictions. The choice of values for the $3+1$ model prediction came from the best fit to the MINOS+ data. 


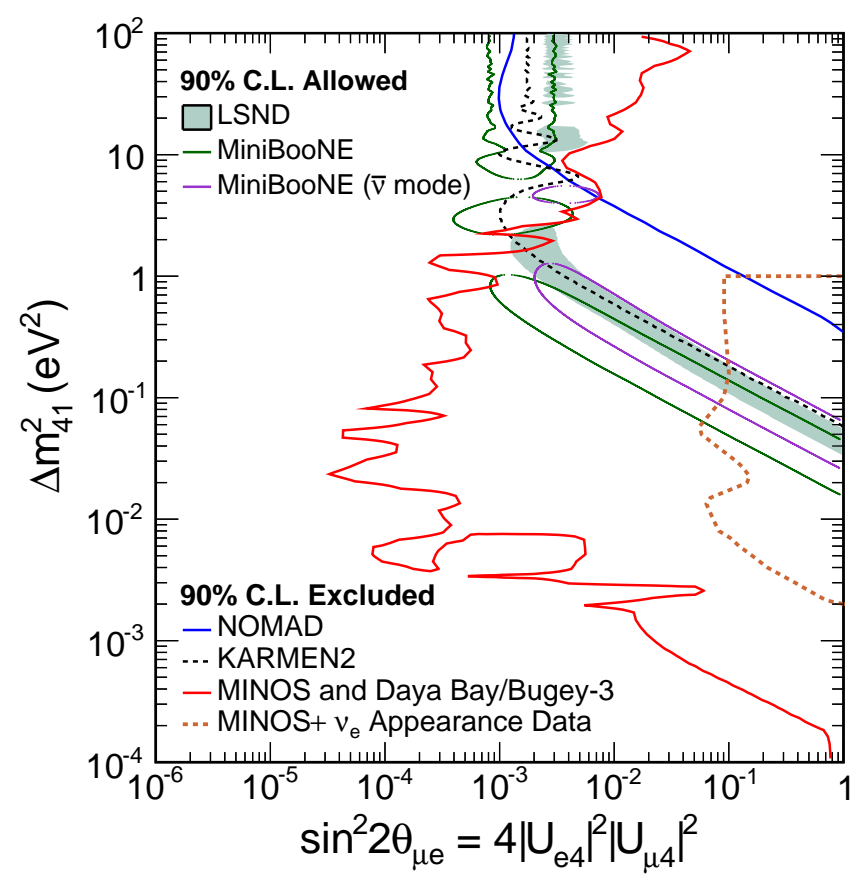

Figure 2: The MINOS+ $90 \%$ C.L. in the $\sin ^{2}\left(2 \theta_{\mu e}\right)$ parameter space over several magnitudes of $\Delta m_{41}^{2}$. The contour for the $v_{e}$-driven analysis is shown (dashed brown) along with results from LSND [2], MiniBooNE [3], the MINOS/Daya Bay/Bugey-3 combination [5], KARMEN2 [6], and NOMAD [7]. Areas to the right of the $90 \%$ C.L. exclusions are disallowed, whereas the LSND and MiniBooNE regions are allowed.

The $v_{\mu} \rightarrow v_{e}$ analysis facilitates a direct comparison to the LSND and MiniBooNE allowed regions. With the first year of MINOS+ data, we increase the tension with LSND and MiniBooNE at values of $\Delta m_{41}^{2}<0.1 \mathrm{eV}^{2}$.

\section{Acknowledgments}

This work was supported by the U.S. DOE; the U.K. STFC; the U.S. NSF; the State and University of Minnesota; and Brazil's FAPESP, CNPq and CAPES. We thank the Minnesota DNR, the Soudan Underground Lab crew, Fermilab, and the TACC for their contributions.

\section{References}

[1] P. Adamson et al. (MINOS), Nucl. Instr. and Meth. A 806, 279 [physics.acc-ph/1507.06690].

[2] A. Aguilar et al. (LSND), Phys. Rev. D 64, 112007 [hep-ex/0104049].

[3] A.A. Aguilar-Arevalo et al. (MiniBooNE), Phys. Rev. Lett. 110, 161801 [hep-ex/1303.2588].

[4] P. Adamson et al. (MINOS), Phys. Rev. Lett. 110, 171801 [hep-ex/1301.4581].

[5] P. Adamson et al. (Daya Bay, MINOS), Phys. Rev. Lett. 117, 151801 [hep-ex/1607.01177].

[6] B. Armbruster et al. (KARMEN2), Phys. Rev. D 65, 112001 [hep-ex/0203021].

[7] P. Astier et al. (NOMAD), Phys. Lett. B 570, 19 [hep-ex/0306037]. 